$\Rightarrow$ CIRCADIAN RHYTHMS

\section{Benefits of time-restricted feeding}

New research utilizing timerestricted feeding (TRF) now shows that metabolic homeostasis is maintained as a result of the circadian clock's control of daily rhythms in feeding and fasting.

Amandine Chaix and colleagues used three different mouse models of altered circadian rhythms: wholebody knockout of Cry1 and Cry2; liver-specific knockout of Bmal1; and liver-specific knockout of $N r 1 d 1$ and $N r 1 d 2$ (also known as $R e v$-erb $\alpha$ and $R e v$-erb $\beta$ ). The mice were fed a high-fat diet either ad libitum or under TRF ( 9 or 10 hours during the dark phase); mice in both groups ate the same amount of calories.

Under ad libitum conditions, the control and mutant mice gained weight and exhibited metabolic abnormalities. Importantly, the mutant mice on TRF were protected from weight gain and metabolic abnormalities. The researchers also conducted extensive transcriptome and metabolome analyses, which showed that TRF reduced the accumulation of lipids in the liver and enhanced cellular defences against metabolic stress.

In addition, the mutant mice on an ad libitum diet were found to have an inflexible respiratory exchange ratio, suggesting that the mice were unable to switch between using carbohydrates and fats for fuel. By contrast, mutant mice on TRF showed normal diurnal patterns in the respiratory exchange ratio.

"Our study shows that TRF protects clock mutant mice from obesity, fatty liver, dyslipidaemia and glucose intolerance without changes in activity or caloric intake," explains corresponding author Satchidananda Panda. "TRF restores rhythms in feeding and fasting, metabolic pathways and nutrient-sensing pathways."

The authors note that their study raises many questions that

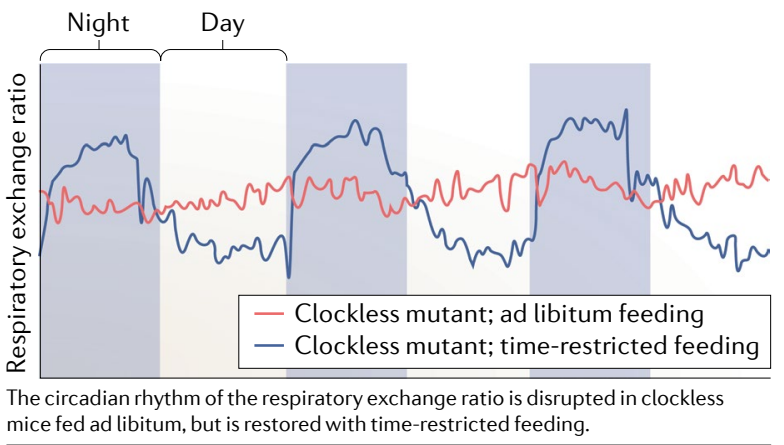

need to be answered. For instance, how the circadian clock regulates feeding rhythms is unclear and the effects of TRF in old animals and over long periods of time are unknown. "Nevertheless, the impact of TRF in delaying onset of metabolic diseases in circadian mutant mice will have significant mechanistic and translational impact," concludes Panda.

Claire Greenhill

ORIGINAL ARTICLE Chaix, A. et al. Time-restricted feeding prevents obesity and metabolic syndrome in mice lacking a circadian clock. Cell Metab. https://doi.org/10.1016/j.cmet.2018.08.004 (2018)

\section{TRF protects clock mutant mice from obesity ...} without changes in activity or caloric intake

OBESITY

\title{
Mechanism revealed for celastrol's effects on weight
}

Celastrol, which is derived from the Thunder God vine (Tripterygium wilfordii; pictured), causes weight loss in animal models of obesity; however, the mechanisms underlying its effects have been unclear. Now, new research published in Diabetes suggests that celastrol induces hypophagia, which results in weight loss.

The researchers administered celastrol once daily for 6 days to mice with high-fat diet (HFD)-induced obesity and chow-fed mice that were not obese. They confirmed previous findings that celastrol results in reduced body weight, fat mass and lean mass in both groups of mice. They also demonstrated that celastrol reduced food intake in the HFD-fed mice, and a small reduction in food intake was also seen in the chow-fed mice.

Next, the researchers treated chow-fed leptin receptor-deficient 8-week-old mice (Lep ${ }^{\mathrm{db}}$ ) and 14-week-old leptin-deficient mice $\left(\right.$ Lep $\left.^{\mathrm{ob}}\right)$ with celastrol. Body weight, fat mass and lean mass were unaffected in these mice, but decreased body weight, fat mass and lean mass were seen in wild-type controls that were fed chow and were treated with celastrol.

The investigators also tested the role of thermogenesis in celastrol's effects on weight loss by assessing the expression levels of genes with protein products involved in thermogenesis. They found that celastrol upregulated the expression of Mcad and Ucp1 in brown adipose tissue and $H s f 1$ and Ucp1 in inguinal white adipose tissue. The expression levels of other key thermogenic genes (such as Pgc1 $\alpha$ ), however, were unaffected in these tissues. In addition, even though UCP1 levels were elevated in adipose tissue, celastrol treatment did not affect energy expenditure or basal metabolic rate, which suggests that thermogenesis was not affected by celastrol.

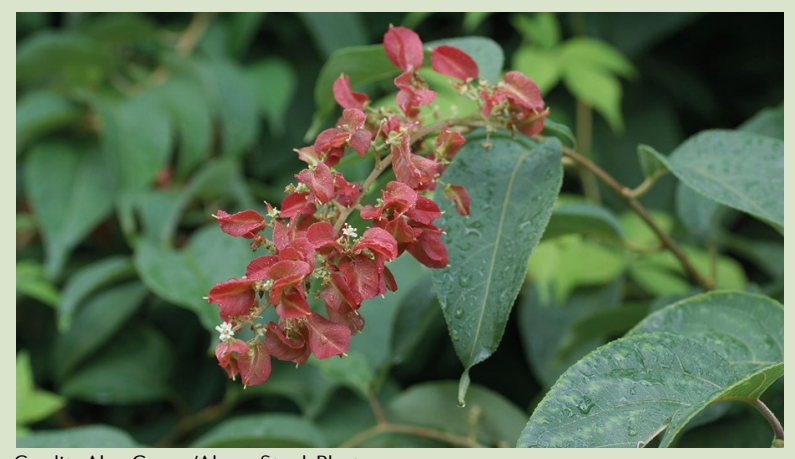

Credit: Alan Gregg/Alamy Stock Photo

The authors conclude that thermogenesis is not a key factor in the weight loss observed with celastrol, and instead suggest that celastrol reduces food intake, which results in weight loss. They note that celastrol was safe and efficacious in their animal models, which supports the further exploration of celastrol reduces food intake, which results in weight loss leptin-sensitizers such as celastrol in the development of new therapeutics for metabolic diseases.

Claire Greenhill

ORIGINAL ARTICLE Pfuhlmann, K. et al. Celastrol induced weight loss is driven by hypophagia and independent from UCP1. Diabetes https:// doi.org/10.2337/db18-0146 (2018) 